\title{
In Situ TEM Investigation on the Thermal Stability of Hydroxyapatite Nanobelts
}

Mei-li Qi ${ }^{1,2}$, Shengkun Yao ${ }^{3}$, Zhennan Huang ${ }^{4}$, Yu-peng $\mathrm{Lu}^{2}$ and Tolou Shokuhfar ${ }^{4}$

${ }^{1}$ School of Transportation Civil Engineering, Shandong Normal University, Ji'nan, Shandong, China (People's Republic), ${ }^{2}$ School of Materials Science and Engineering, Shandong University, Ji'nan, Shandong, China (People's Republic), ${ }^{3}$ School of Physics and Electronics, Shandong Normal University, Ji'nan, Shandong, China (People's Republic), ${ }^{4}$ University of Illinois at Chicago, Chicago, Illinois, United States

*Corresponding author: tolou@uic.edu, biosdu@sdu.edu.cn

The thermal stability of hydroxyapatite (HA) nanobelts can directly influence the mechanical and biological properties of HA reinforced composites. ${ }^{1}$ Therefore, heat treatments are always involved in preparing those composites. The decomposition of HA partly into $\beta$-tricalcium phosphate $(\beta$-TCP) when heated to a high temperature has been reported. ${ }^{1,2}$ However, many things remain unclear since we cannot get the dynamic transformation process by using traditional methods. Key questions that need to be solved include: (1) How does the HA to $\beta$-TCP transformation occur and develop? (2) What morphology and phase change does the sample experience during the process? These key questions call for a direct microscopy investigation, especially dynamical high resolution imaging on the thermal response of HA nanobelts. Fortunately, in recent years, in situ heating transmission electron microscopy (TEM) has enabled the investigation of such dynamic biological processes at nanoscale. It has been widely used in studies of temperature related material behaviors, such as phase transformations, solid/gas-solid reactions, microstructural changes, growth of nanostructures, sintering of catalysts, et al. ${ }^{3}$ Compared with conventional furnace heating method, in situ heating technique makes the real-time observation of microstructural evolution and phase transformation process during heating come true. Here, we report the first in situ TEM study on the thermal stability of HA nanobelts at elevated temperatures. We demonstrate the phase transition from HA to $\beta$-TCP and microstructural evolution in the continuous heating process. In the experiments, both the morphology and corresponding diffraction patterns are captured under room temperature, $200^{\circ} \mathrm{C}, 400^{\circ} \mathrm{C}, 600^{\circ} \mathrm{C}, 800^{\circ} \mathrm{C}, 850^{\circ} \mathrm{C}, 900^{\circ} \mathrm{C}$, respectively. As we expected, HA nanobelts are stable at relatively high temperatures (below $800^{\circ} \mathrm{C}$ ) and no phase transition and structural deterioration was detected. When we further increase the heating temperature, $\beta$-TCP phase is detected. Meanwhile, the building units appeared to be sintered together. The direct evidence can be recorded by in situ TEM. This new understanding is important to address the microstructural evolution and phase transformation of apatite products, such as select optimum heat treatment parameters to meet different performance requirements. ${ }^{4}$

\section{References}

[1] Raynaud S, et al. Biomaterials 23 (2002), p. 1065-1072.

[2] Qi M, et al. Surf Innov 5 (2017), p. 75-81.

[3] Sharifi-Asl S, et al. Nano Lett. 17 (2017), p. 2165-2171.

[4] The authors acknowledge funding from the Doctoral Scientific Research Foundation of Shandong Jiaotong University (Award No. 305-50004919), National Science Foundation of China (Award No. 11947117), and Postdoctoral Science Foundation of China (2019M660164). We also thank UIC Research Resources Center for assisting the usage of their equipment and instrumentation. 